\title{
Generalized Inequalities Related to the Classical Euler's Gamma Function
}

\author{
Kwara Nantomah* \\ Department of Mathematics, University for Development Studies, Navrongo Campus, Navrongo UE/R, Ghana \\ *Corresponding author: mykwarasoft@yahoo.com, knantomah@uds.edu.gh \\ Received October 10, 2014; Revised November 26, 2014; Accepted December 14, 2014
}

\begin{abstract}
This paper presents some inequalities concerning certain ratios of the classical Euler's Gamma function. The results generalized some recent results.
\end{abstract}

Keywords: Gamma function, q-Gamma function, k-Gamma function, (p,q)-Gamma function, (q,k)-Gamma function, inequality

Cite This Article: Kwara Nantomah, "Generalized Inequalities Related to the Classical Euler's Gamma Function.” Turkish Journal of Analysis and Number Theory, vol. 2, no. 6 (2014): 226-229. doi: 10.12691/tjant-2-6-7.

\section{Introduction}

We begin by outlining the following basic definitions well-known in literature.

The celebrated classical Euler's Gamma function, $\Gamma(t)$ is defined for $t>0$ as

$$
\Gamma(t)=\int_{0}^{\infty} e^{-x} x^{t-1} d x .
$$

The q-Gamma function, $\Gamma_{q}(t)$ is defined for $q \in(0,1)$ and $t>0$ as (see [2])

$$
\Gamma_{q}(t)=(1-q)^{1-t} \prod_{n=1}^{\infty} \frac{1-q^{n}}{1-q^{t+n}} .
$$

Also, the k-Gamma function, $\Gamma_{k}(t)$ was defined by Diaz and Pariguan [1] for $k>0$ and $t>0$ as

$$
\Gamma_{k}(t)=\int_{0}^{\infty} e^{-\frac{x^{k}}{k}} x^{t-1} d x .
$$

Diaz and Teruel [5] further defined the (q,k)-Gamma function $\Gamma_{(q, k)}(t)$ for $q \in(0,1), k>0$ and $t>0$ as

$$
\Gamma_{(q, k)}(t)=\frac{\left(1-q^{k}\right)_{q, k}^{\frac{t}{k}-1}}{(1-q)^{\frac{t}{k}-1}},
$$

where

$$
\begin{aligned}
(t)_{n, k} & =t(t+k)(t+2 k) \cdots(t+(n-1) k) \\
& =\prod_{j=0}^{n-1}(t+j k)
\end{aligned}
$$

is the k-generalized Pochhammer symbol.

Furthermore, Krasniqi and Merovci [4] defined the $(\mathrm{p}, \mathrm{q})$-Gamma function $\Gamma_{(p, q)}(t)$ for $p \in N, q \in(0,1)$ and $t>0$ as

$$
\Gamma_{(p, q)}(t)=\frac{[p]_{q}^{t}[p]_{q} !}{[t]_{q}[t+1]_{q} \cdots[t+p]_{q}},
$$

where

$$
[p]_{q}=\frac{1-q^{p}}{1-q} .
$$

The psi function, $\psi(t)$ otherwise known as the digamma function is defined as the logarithmic derivative of the Gamma function. That is,

$$
\psi(t)=\frac{d}{d t} \operatorname{In} \Gamma(t)=\frac{\Gamma^{\prime}(t)}{\Gamma(t)} .
$$

The q-digamma function, $\mathrm{k}$-digamma function, $(\mathrm{p}, \mathrm{q})$ diagamma function and (q,k)-digamma function are similarly defined as follows:

$$
\begin{aligned}
& \psi_{q}(t)=\frac{d}{d t} \operatorname{In} \Gamma_{q}(t)=\frac{\Gamma_{q}^{\prime}(t)}{\Gamma_{q}(t)}, \\
& \psi_{k}(t)=\frac{d}{d t} \operatorname{In} \Gamma_{k}(t)=\frac{\Gamma_{k}^{\prime}(t)}{\Gamma_{k}(t)}, \\
& \psi_{(p, q)}(t)=\frac{d}{d t} \operatorname{In} \Gamma_{(p, q)}(t)=\frac{\Gamma_{(p, q)}^{\prime}(t)}{\Gamma_{(p, q)}(t)}
\end{aligned}
$$

and

$$
\psi_{(q, k)}(t)=\frac{d}{d t} \operatorname{In} \Gamma_{(q, k)}(t)=\frac{\Gamma_{(q, k)}^{\prime}(t)}{\Gamma_{(q, k)}(t)} .
$$

It is common knowledge that these functions exhibit the following series charaterizations (see also [7-12]): 


$$
\begin{gathered}
\psi_{q}(t)=-\operatorname{In}(1-q)+(\operatorname{In} q) \sum_{n=1}^{\infty} \frac{q^{n t}}{1-q^{n}} \\
\psi_{k}(t)=\frac{\operatorname{In} k-\gamma}{k}-\frac{1}{t}+\sum_{n=1}^{\infty} \frac{t}{n k(n k+t)} \\
\psi_{(p, q)}(t)=\operatorname{In}[p]_{q}+(\operatorname{In} q) \sum_{n=1}^{p} \frac{q^{n t}}{1-q^{n}} \\
\psi_{(q, k)}(t)=\frac{-\operatorname{In}(1-q)}{k}+(\operatorname{In} q) \sum_{n=1}^{\infty} \frac{q^{n k t}}{1-q^{n k}}
\end{gathered}
$$

where $\gamma=0.577215664901532 \ldots$ represents the EulerMascheroni's constant.

Of late, the following double inequalities were presented in [7] by the use of some monotonicity properties of some functions related with the Gamma function.

$$
\begin{aligned}
\frac{(1-q)^{-t} \Gamma_{q}(\alpha)}{[p]_{q}^{t} \Gamma_{(p, q)}(\alpha)} & \geq \frac{\Gamma_{q}(\alpha+t)}{\Gamma_{(p, q)}(\alpha+t)} \\
& \geq \frac{(1-q)^{1-t} \Gamma_{q}(\alpha+1)}{[p]_{q}^{t-1} \Gamma_{(p, q)}(\alpha+1)}
\end{aligned}
$$

for $t \in(0,1), \alpha>0, p \in N$ and $q \in(0,1)$.

$$
\begin{array}{r}
\frac{(1-q)^{-t} \Gamma_{q}(\alpha)}{(1-q)^{-\frac{t}{k}} \Gamma_{(q, k)}(\alpha)} \geq \frac{\Gamma_{q}(\alpha+t)}{\Gamma_{(q, k)}(\alpha+t)} \\
\geq \frac{(1-q)^{1-t} \Gamma_{q}(\alpha+1)}{(1-q)^{\frac{1}{k}(1-t)} \Gamma_{(q, k)}(\alpha+1)}
\end{array}
$$

for $t \in(0,1), \alpha>0, q \in(0,1)$ and $k \geq 1$.

$$
\begin{array}{r}
\frac{\alpha k^{\frac{t}{k}} e^{-\frac{\gamma t}{k}} \Gamma_{k}(\alpha)}{(\alpha+t)[p]_{q}^{t} \Gamma_{(p, q)}(\alpha)}<\frac{\Gamma_{k}(\alpha+t)}{\Gamma_{(p, q)}(\alpha+t)} \\
<\frac{(\alpha+1) k^{\frac{t-1}{k}} e^{\frac{\gamma(1-t)}{k}} \Gamma_{k}(\alpha+1)}{(\alpha+t)[p]_{q}^{t-1} \Gamma_{(p, q)}(\alpha+1)}
\end{array}
$$

for $t \in(0,1), \alpha>0, p \in N, q \in(0,1)$ and $k>0$.

$$
\begin{gathered}
\frac{\alpha k^{\frac{t}{k}} e^{-\frac{\gamma t}{k}} \Gamma_{k}(\alpha)}{(\alpha+t)(1-q)^{-\frac{t}{k}} \Gamma_{(q, k)}(\alpha)}<\frac{\Gamma_{k}(\alpha+t)}{\Gamma_{(q, k)}(\alpha+t)} \\
<\frac{\alpha k^{\frac{t}{k}} e^{-\frac{\gamma t}{k}} \Gamma_{k}(\alpha+1)}{(\alpha+t)(1-q)^{\frac{1-t}{k}} \Gamma_{(q, k)}(\alpha+1)}
\end{gathered}
$$

for $t \in(0,1), \alpha>0, q \in(0,1)$ and $k>0$.

Results of this form can also be found in [8,9,10,11,12]. By utilizing similar techniques as in the previous results, this paper seeks to provide some generalizations of the above inequalities. We present our results in the following sections.

\section{Supporting Results}

We begin with the following Lemmas.

Lemma 2.1. Suppose that $\alpha>0, \beta>0$, $u \geq w>0, t>0, p \in N$ and $q \in(0,1)$. Then,

$$
\begin{aligned}
& u \operatorname{In}(1-q)+w \operatorname{In}[p]_{q} \\
& +u \psi_{q}(\alpha+\beta t)-w \psi_{(p, q)}(\alpha+\beta t) \leq 0 .
\end{aligned}
$$

Proof. From the characterization in equations (1) and (3) we obtain,

$$
\begin{aligned}
& u \operatorname{In}(1-q)+w \operatorname{In}[p]_{q}+u \psi_{q}(t)-w \psi(p, q) \\
& =(\operatorname{In} q)\left[u \sum_{n=1}^{\infty} \frac{q^{n t}}{1-q^{n}}-w \sum_{n=1}^{p} \frac{q^{n t}}{1-q^{n}}\right] \leq 0
\end{aligned}
$$

We conclude the proof by substituting $t$ by $\alpha+\beta t$.

Lemma 2.2. Suppose that $\alpha>0, \beta>0$, $u \geq w>0, t>0, q \in(0,1)$ and $k \geq 1$. Then,

$$
\begin{aligned}
& u \operatorname{In}(1-q)-w \frac{\operatorname{In}(1-q)}{k} \\
+ & u \psi_{q}(\alpha+\beta t)-w \psi_{(q, k)}(\alpha+\beta t) \leq 0 .
\end{aligned}
$$

Proof. From the characterization in equations (1) and (4) we obtain,

$$
\begin{aligned}
& u \operatorname{In}(1-q)-w \frac{\operatorname{In}(1-q)}{k}+u \psi_{q}(t)-w \psi_{(q, k)}(t) \\
= & (\operatorname{In} q) \sum_{n=1}^{\infty}\left[u \frac{q^{n t}}{1-q^{n}}-w \frac{q^{n k t}}{1-q^{n k}}\right] \leq 0 .
\end{aligned}
$$

We conclude the proof by substituting $t$ by $\alpha+\beta t$.

Lemma 2.3. Suppose that $\alpha>0, \beta>0, u>0 \quad w>0$, $t>0, k>0, p \in N$ and $q \in(0,1)$. Then,

$$
\begin{gathered}
w \operatorname{In}[p]_{q}-u \frac{\operatorname{In} k}{k}+\frac{u \gamma}{k}+\frac{u}{\alpha+\beta t} \\
+u \psi_{k}(\alpha+\beta t)-w \psi_{(p, q)}(\alpha+\beta t)>0 .
\end{gathered}
$$

Proof. From the characterization in equations (2) and (3) we obtain,

$$
\begin{aligned}
& w \operatorname{In}[p]_{q}-u \frac{\operatorname{In} k}{k}+\frac{u \gamma}{k}+\frac{u}{t}+u \psi_{q}(t)-w \psi_{(p, q)}(t) \\
= & u \sum_{n=1}^{\infty} \frac{t}{n k(n k+t)}-w \sum_{n=1}^{p} \frac{q^{n t}}{1-q^{n}}>0 .
\end{aligned}
$$

We conclude the proof by substituting $t$ by $\alpha+\beta t$.

Lemma 2.4. Suppose that $\alpha>0, \beta>0, u>0 \quad w>0$, $t>0, q \in(0,1)$ and $k>0$. Then,

$$
\begin{aligned}
& -\frac{\operatorname{In}\left(k^{u}(1-q)^{w}\right)}{k}+\frac{u \gamma}{k}+\frac{u}{\alpha+\beta t} \\
& +u \psi_{k}(\alpha+\beta t)-w \psi_{(q, k)}(\alpha+\beta t)>0 .
\end{aligned}
$$

Proof. From the characterization in equations (2) and (4) we obtain, 


$$
\begin{aligned}
& -\frac{\operatorname{In}\left(k^{u}(1-q)^{w}\right)}{k}+\frac{u \gamma}{k}+\frac{u}{\alpha+\beta t}+u \psi_{q}(t)-w \psi_{(q, k)}(t) \\
& =u \sum_{n=1}^{\infty} \frac{t}{n k(n k+t)}-w(\operatorname{In} q) \sum_{n=1}^{\infty} \frac{q^{n k t}}{1-q^{n k}}>0 .
\end{aligned}
$$

We conclude the proof by substituting $t$ by $\alpha+\beta t$.

\section{Main Results}

We now present our results in the following Theorems.

Theorem 3.1. Define a function $E$ for $p \in N$ and $q \in(0,1)$ by

$$
E(t)=\frac{(1-q)^{u \beta t} \Gamma_{q}(\alpha+\beta t)^{u}}{[p]_{q}^{-w \beta t} \Gamma_{(p, q)}(\alpha+\beta t)^{w}}, t \in(0, \infty)
$$

where $u, w, \alpha, \beta$ are positive real numbers such that $u \geq w$. Then, $E$ is non-increasing on $t \in(0, \infty)$ and the inequalities:

$$
\begin{array}{r}
\frac{(1-q)^{-u \beta t} \Gamma_{q}(\alpha)^{u}}{[p]_{q}^{w \beta t} \Gamma_{(p, q)}(\alpha)^{w}} \geq \frac{\Gamma_{q}(\alpha+\beta t)^{u}}{\Gamma_{(p, q)}(\alpha+\beta t)^{w}} \\
\geq \frac{(1-q)^{u \beta(1-t)} \Gamma_{q}(\alpha+\beta)^{u}}{[p]_{q}^{w \beta(t-1)} \Gamma_{(p, q)}(\alpha+\beta)^{w}}
\end{array}
$$

are valid for each $t \in(0,1)$.

Proof. Let $\lambda(t)=\operatorname{In} E(t)$ for every $t \in(0, \infty)$. Then

$$
\begin{aligned}
& \lambda(t)=\operatorname{In} \frac{(1-q)^{u \beta t} \Gamma_{q}(\alpha+\beta t)^{u}}{[p]_{q}^{-w \beta t} \Gamma_{(p, q)}(\alpha+\beta t)^{w}} \\
& =u \beta t \operatorname{In}(1-q)+w \beta t \operatorname{In}[p]_{q} \\
& \quad+u \operatorname{In} \Gamma_{q}(\alpha+\beta t)-w \operatorname{In} \Gamma_{(p, q)}(\alpha+\beta t)
\end{aligned}
$$

Then,

$$
\begin{aligned}
& \lambda^{\prime}(t)=u \beta \operatorname{In}(1-q)+w \beta \operatorname{In}[p]_{q} \\
& \quad+u \beta \psi_{q}(\alpha+\beta t)-w \beta \psi_{(p, q)}(\alpha+\beta t) \\
& =\beta\left[u \operatorname{In}(1-q)+w \operatorname{In}[p]_{q}\right. \\
& \quad+u \psi_{q}(\alpha+\beta t)-w \psi(p, q) \\
& \quad(\alpha+\beta t)] \leq 0
\end{aligned}
$$

as a result of Lemma 2.1. That implies $\lambda$ is nonincreasing on $t \in(0, \infty)$. Consequently, $E$ is nonincreasing on $t \in(0, \infty)$ and for each $t \in(0,1)$ we have,

$$
E(0) \geq E(t) \geq E(1)
$$

yielding equation (10).

Theorem 3.2. Define a function $F$ for $q \in(0,1)$ and $k \geq 1$ by

$$
F(t)=\frac{(1-q)^{u \beta t} \Gamma_{q}(\alpha+\beta t)^{u}}{(1-q)^{\frac{u \beta t}{k}} \Gamma_{(q, k)}(\alpha+\beta t)^{w}}, t \in(0, \infty)
$$

where $u, w, \alpha, \beta$ are positive real numbers such that $u \geq w$. Then, $F$ is non-increasing on $t \in(0, \infty)$ and the inequalities:

$$
\begin{array}{r}
\frac{(1-q)^{-u \beta t} \Gamma_{q}(\alpha)^{u}}{(1-q)^{-\frac{w \beta t}{k}} \Gamma_{(q, k)}(\alpha)^{w}} \geq \frac{\Gamma_{q}(\alpha+\beta t)^{u}}{\Gamma_{(q, k)}(\alpha+\beta t)^{w}} \\
\geq \frac{(1-q)^{u \beta(1-t)} \Gamma_{q}(\alpha+\beta)^{u}}{(1-q)^{\frac{w \beta}{k}(1-t)} \Gamma_{(q, k)}(\alpha+\beta)^{w}}
\end{array}
$$

are valid for each $t \in(0,1)$.

Proof. Let $\eta(t)=\operatorname{In} F(t)$ for every $t \in(0, \infty)$. Then

$$
\begin{aligned}
& \eta(t)=\operatorname{In} \frac{(1-q)^{u \beta t} \Gamma_{q}(\alpha+\beta t)^{u}}{(1-q)^{\frac{u \beta t}{k}} \Gamma_{(q, k)}(\alpha+\beta t)^{w}} \\
& =u \beta t \operatorname{In}(1-q)-\frac{w \beta t}{k} \operatorname{In}(1-q) \\
& +u \operatorname{In} \Gamma_{q}(\alpha+\beta t)-w \operatorname{In} \Gamma_{(q, k)}(\alpha+\beta t)
\end{aligned}
$$

Then,

$$
\begin{aligned}
& \eta^{\prime}(t)=u \beta \operatorname{In}(1-q)-\frac{w \beta}{k} \operatorname{In}(1-q) \\
& +u \beta \psi_{q}(\alpha+\beta t)-w \beta \psi_{(q, k)}(\alpha+\beta t) \\
& =\beta\left[u \operatorname{In}(1-q)-w \frac{\operatorname{In}(1-q)}{k}\right. \\
& \left.\quad+u \psi_{q}(\alpha+\beta t)-w \psi_{(q, k)}(\alpha+\beta t)\right] \leq 0
\end{aligned}
$$

as a result of Lemma 2.2. That implies $\eta$ is nonincreasing on $t \in(0, \infty)$. Consequently, $F$ is nonincreasing on $t \in(0, \infty)$ and for each $t \in(0,1)$ we have,

$$
F(0) \geq F(t) \geq F(1)
$$

yielding equation (12).

Theorem 3.3. Define a function $G$ for $t \in(0, \infty)$, $p \in N, q \in(0,1)$ and $k>0$ by

$$
G(t)=\frac{(\alpha+\beta t)^{u} k^{-\frac{u \beta t}{k}} e^{\frac{u \beta \gamma t}{k}} \Gamma_{k}(\alpha+\beta t)^{u}}{[p]_{q}^{-w \beta t} \Gamma_{(p, q)}(\alpha+\beta t)^{w}}
$$

where $u, w, \alpha, \beta$ are positive real numbers. Then, $G$ is increasing on $t \in(0, \infty)$ and the inequalities:

$$
\begin{aligned}
& \frac{\alpha^{u} k^{\frac{u \beta t}{k}} e^{-\frac{u \beta \gamma t}{k}} \Gamma_{k}(\alpha)^{u}}{(\alpha+\beta t)^{u}[p]_{q}^{w \beta t} \Gamma_{(p, q)}(\alpha)^{w}} \\
& <\frac{\Gamma_{k}(\alpha+\beta t)^{u}}{\Gamma_{(p, q)}(\alpha+\beta t)^{w}} \\
& <\frac{(\alpha+\beta)^{u} k^{\frac{u \beta(t-1)}{k}} e^{\frac{u \beta \gamma(1-t)}{k}} \Gamma_{k}(\alpha+\beta)^{u}}{(\alpha+\beta t)^{u}[p]_{q}^{w \beta(t-1)} \Gamma_{(p, q)}(\alpha+\beta)^{w}}
\end{aligned}
$$

are valid for each $t \in(0,1)$.

Proof. Let $\mu(t)=\operatorname{In} G(t)$ for every $t \in(0, \infty)$. Then

$$
\mu(t)=\operatorname{In} \frac{(\alpha+\beta t)^{u} k^{-\frac{u \beta t}{k}} e^{\frac{u \beta \gamma t}{k}} \Gamma_{k}(\alpha+\beta t)^{u}}{[p]_{q}^{-w \beta t} \Gamma_{(p, q)}(\alpha+\beta t)^{w}}
$$




$$
\begin{aligned}
= & u \operatorname{In}(\alpha+\beta t)-\frac{u \beta t}{k} \operatorname{In} k+\frac{u \beta \gamma t}{k}+w \beta t \operatorname{In}[p]_{q} \\
& +u \operatorname{In} \Gamma_{k}(\alpha+\beta t)-w \operatorname{In} \Gamma_{(p, q)}(\alpha+\beta t) .
\end{aligned}
$$

Then,

$$
\begin{aligned}
& \mu^{\prime}(t)=w \beta \operatorname{In}[p]_{q}-u \beta \frac{\operatorname{In} k-\gamma}{k}+\frac{u \beta}{\alpha+\beta t} \\
& +u \beta \psi_{k}(\alpha+\beta t)-w \beta \psi_{(p, q)}(\alpha+\beta t) \\
& =\beta\left[w \operatorname{In}[p]_{q}-u \frac{\operatorname{In} k}{k}+\frac{u \gamma}{k}+\frac{u}{\alpha+\beta t}\right. \\
& \left.\quad+u \psi_{k}(\alpha+\beta t)-w \psi_{(p, q)}(\alpha+\beta t)\right]>0
\end{aligned}
$$

as a result of Lemma 2.3. That implies $\mu$ is nonincreasing on $t \in(0, \infty)$. Consequently, $G$ is nonincreasing on $t \in(0, \infty)$ and for each $t \in(0,1)$ we have,

$$
G(0)<G(t)<G(1)
$$

yielding equation (14).

Theorem 3.4. Define a function $H$ for $t \in(0, \infty)$, $q \in(0,1)$ and $k>0$ by

$$
H(t)=\frac{(\alpha+\beta t)^{u} e^{\frac{u \beta \gamma t}{k}} \Gamma_{k}(\alpha+\beta t)^{u}}{k^{\frac{u \beta t}{k}}(1-q)^{\frac{w \beta t}{k}} \Gamma_{(q, k)}(\alpha+\beta t)^{w}}
$$

where $u, w, \alpha, \beta$ are positive real numbers. Then, $H$ is increasing on $t \in(0, \infty)$ and the inequalities:

$$
\begin{gathered}
\frac{\alpha^{u} e^{-\frac{u \beta \gamma t}{k}} \Gamma_{k}(\alpha)^{u}}{(\alpha+\beta t)^{u} k^{-\frac{u \beta t}{k}}(1-q)^{-\frac{w \beta t}{k}} \Gamma_{(q, k)}(\alpha)^{w}} \\
<\frac{\Gamma_{k}(\alpha+\beta t)^{u}}{\Gamma_{(q, k)}(\alpha+\beta t)^{w}} \\
<\frac{(\alpha+\beta)^{u} e^{\frac{u \beta \gamma(1-t)}{k}} \Gamma_{k}(\alpha+\beta)^{u}}{(\alpha+\beta t)^{u} k^{\frac{u \beta(1-t)}{k}}(1-q)^{\frac{w \beta(1-t)}{k}} \Gamma_{(q, k)}(\alpha+\beta)^{w}}
\end{gathered}
$$

are valid for each $t \in(0,1)$.

Proof. Let $\delta(t)=\operatorname{In} H(t)$ for every $t \in(0, \infty)$. Then

$$
\begin{aligned}
& \delta(t)=\operatorname{In} \frac{(\alpha+\beta t)^{u} e^{\frac{u \beta \gamma t}{k}} \Gamma_{k}(\alpha+\beta t)^{u}}{k^{\frac{w \beta t}{k}}(1-q)^{\frac{w \beta t}{k}} \Gamma_{(q, k)}(\alpha+\beta t)^{w}} \\
& =u \operatorname{In}(\alpha+\beta t)+\frac{u \beta \gamma t}{k}-\frac{u \beta t}{k} \operatorname{In} k-\frac{w \beta t}{k} \operatorname{In}(1-q) \\
& +u \operatorname{In} \Gamma_{k}(\alpha+\beta t)-w \operatorname{In} \Gamma_{(q, k)}(\alpha+\beta t) .
\end{aligned}
$$

Then,

$$
\begin{aligned}
& \delta^{\prime}(t)=-\beta \frac{\operatorname{In}\left(k^{u}(1-q)^{w}\right)}{k}+\frac{u \beta \gamma}{k}+\frac{u \beta}{\alpha+\beta t} \\
& +u \beta \psi_{k}(\alpha+\beta t)-w \beta \psi_{(q, k)}(\alpha+\beta t) \\
& =\beta\left[-\frac{\operatorname{In}\left(k^{u}(1-q)^{w}\right)}{k}+\frac{u \gamma}{k}+\frac{u}{\alpha+\beta t}\right. \\
& \left.\quad+u \psi_{k}(\alpha+\beta t)-w \psi_{(q, k)}(\alpha+\beta t)\right]>0
\end{aligned}
$$

as a result of Lemma 2.4. That implies $\delta$ is nonincreasing on $t \in(0, \infty)$. Consequently, $H$ is nonincreasing on $t \in(0, \infty)$ and for each $t \in(0,1)$ we have,

$$
H(0)<H(t)<H(1)
$$

yielding equation (16).

\section{Conclusion}

If we fix $u=w=\beta=1$ in inequalities (10), (12), (14) and (16), then we respectively obtain the inequalities (5), (6), (7) and (8) as special cases. By this, the previous results [7] have been generalized.

\section{Competing Interests}

The authors have no competing interests.

\section{References}

[1] R. Diaz and E. Pariguan, On hypergeometric functions and Pachhammer k-symbol, Divulgaciones Matematicas 15(2)(2007), 179-192.

[2] T. Mansour, Some inequalities for the q-Gamma Function, J. Ineq. Pure Appl. Math. 9(1)(2008), Art. 18.

[3] F. Merovci, Power Product Inequalities for the $\Gamma_{k}$ Function, Int. Journal of Math. Analysis, 4(21)(2010), 1007-1012.

[4] V. Krasniqi and F. Merovci, Some Completely Monotonic Properties for the (p, q)-Gamma Function, Mathematica Balkanica, New Series 26(2012), 1-2.

[5] R. Diaz and C. Teruel, q,k-generalized gamma and beta functions, J. Nonlin. Math. Phys. 12(2005), 118-134.

[6] V. Krasniqi, T. Mansour and A. Sh. Shabani, Some Monotonicity Properties and Inequalities for $\Gamma$ and $\zeta$ Functions, Mathematical Communications 15(2)(2010), 365-376.

[7] K. Nantomah, On Certain Inequalities Concerning the Classical Euler's Gamma Function, Advances in Inequalities and Applications, Vol. 2014 (2014) Art ID 42.

[8] K. Nantomah and M. M. Iddrisu, Some Inequalities Involving the Ratio of Gamma Functions, Int. Journal of Math. Analysis 8(12)(2014), 555-560.

[9] K. Nantomah, M. M. Iddrisu and E. Prempeh, Generalization of Some Inequalities for theRatio of Gamma Functions, Int. Journal of Math. Analysis, 8(18)(2014), 895-900.

[10] K. Nantomah and E. Prempeh, Generalizations of Some Inequalities for the p-Gamma, q-Gamma and k-Gamma Functions, Electron. J. Math. Anal. Appl. 3(1)(2015),158-163.

[11] K. Nantomah and E. Prempeh, Some Sharp Inequalities for the Ratio of Gamma Functions, Math. Aeterna, 4(5)(2014), 501-507.

[12] K. Nantomah and E. Prempeh, Generalizations of Some Sharp Inequalities for the Ratio of Gamma Functions, Math. Aeterna, 4(5)(2014), 539-544. 